\title{
Application of WHO's guideline for the selection of sentinel sites for hospital-based influenza surveillance in Indonesia
}

Ni Ketut Susilarini ${ }^{*+}$, Martahan Sitorus ${ }^{2 \dagger}$, Catharina Yekti Praptaningsih ${ }^{3}$, Ondri Dwi Sampurno ${ }^{1}$, Arie Bratasena ${ }^{2}$, Ester Mulyadi ${ }^{3}$, Roselinda Rusli ${ }^{1}$, Ahmad Fandil ${ }^{2}$, Amalya Mangiri ${ }^{3}$, Hana Apsari ${ }^{1}$, Edy Hariyanto ${ }^{2}$ and Gina Samaan ${ }^{3^{*}}$

\begin{abstract}
Background: A sentinel hospital-based severe acute respiratory infection (SARI) surveillance system was established in Indonesia in 2013. Deciding on the number, geographic location and hospitals to be selected as sentinel sites was a challenge. Based on the recently published WHO guideline for influenza surveillance (2012), this study presents the process for hospital sentinel site selection.

Methods: From the 2,165 hospitals in Indonesia, the first step was to shortlist to hospitals that had previously participated in respiratory disease surveillance systems and had acceptable surveillance performance history. The second step involved categorizing the shortlist according to five regions in Indonesia to maximize geographic representativeness. A checklist was developed based on the WHO recommended attributes for sentinel site selection including stability, feasibility, representativeness and the availability of data to enable disease burden estimation. Eight hospitals, a maximum of two per geographic region, were visited for checklist administration. Checklist findings from the eight hospitals were analyzed and sentinel sites selected in the third step.

Results: Six hospitals could be selected based on resources available to ensure system stability over a three-year period. For feasibility, all eight hospitals visited had mechanisms for specimen shipment and the capacity to report surveillance data, but two had limited motivation for system participation. For representativeness, the eight hospitals were geographically dispersed around Indonesia, and all could capture cases in all age and socio-economic groups. All eight hospitals had prerequisite population data to enable disease burden estimation. The two hospitals with low motivation were excluded and the remaining six were selected as sentinel sites.

Conclusions: The multi-step process enabled sentinel site selection based on the WHO recommended attributes that emphasize right-sizing the surveillance system to ensure its stability and maximizing its geographic representativeness. This experience may guide other countries interested in adopting WHO's influenza surveillance standards for sentinel site selection.
\end{abstract}

Keywords: Surveillance, Indonesia, Pneumonia, System, Respiratory disease, Severe acute respiratory infection, Influenza-like illness

\footnotetext{
* Correspondence: niketutsusi@yahoo.com; ginasamaan@yahoo.com

${ }^{\dagger}$ Equal contributors

${ }^{1}$ National Institute of Health Research and Development, Ministry of Health, Jakarta, Indonesia

${ }^{3}$ Influenza Division, United States Centers for Disease Control and Prevention, Jakarta, Indonesia

Full list of author information is available at the end of the article
}

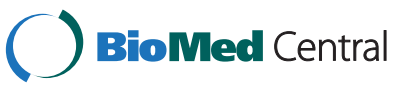

(c) 2014 Susilarini et al.; licensee BioMed Central Ltd. This is an Open Access article distributed under the terms of the Creative Commons Attribution License (http://creativecommons.org/licenses/by/2.0), which permits unrestricted use, distribution, and reproduction in any medium, provided the original work is properly credited. The Creative Commons Public Domain Dedication waiver (http://creativecommons.org/publicdomain/zero/1.0/) applies to the data made available in this article, unless otherwise stated. 


\section{Background}

Influenza surveillance has been instrumental in advancing global understanding of the disease, monitoring changes in antigenicity of different subtypes and guiding influenza vaccine strain selection $[1,2]$. With the emergence and circulation of the avian influenza A/H5N1 virus in several countries, including in Indonesia, influenza surveillance became a global public health priority to prepare for an ensuing influenza pandemic. During the influenza A/H1N1pdm09 pandemic in 2009, influenza surveillance systems enabled monitoring of pandemic disease trends, severity and mortality [3-5].

In July 2012, the World Health Organization (WHO) released updated standards for influenza surveillance [6]. This guideline built on the lessons learnt from the 2009 influenza pandemic and outlined both the epidemiological and virological surveillance objectives, as well as revised case definitions and minimum data set requirements. The primary objective of sentinel influenza surveillance systems as stated in the guideline is to monitor influenza activity rather than to detect outbreaks. Sentinel influenza surveillance systems can provide valuable information about epidemiological trends including seasonality, age groups affected and groups at risk of severe disease. Sentinel systems can also provide information about virological trends, and to identify the emergence of viruses with unique characteristics or with antigenic drifts or shifts [7].

As of September 2012, Indonesia had seven surveillance systems administered by Ministry of Health $(\mathrm{MOH})$ that provided information about acute respiratory illness (ARI), pneumonia and influenza (Table 1). Three of these systems had hospital-based surveillance sites. Evaluations of two hospital-based sentinel surveillance systems, the sentinel pneumonia surveillance system operated by the ARI Subdirectorate since 2007 and the laboratory-based severe acute respiratory infection (SARI) surveillance system operated by the National Institute of Health Research and Development (NIHRD) since 2006, found that both had limited utility. Both systems had sites with very low surveillance activity and the sentinel pneumonia surveillance system lacked laboratory data while the SARI surveillance system lacked epidemiological focus. This led the ARI Subdirectorate and NIHRD teams to terminate these systems in December 2012 and combine efforts to establish a SARI surveillance system in 2013 that addressed their collective needs.

The new SARI system called 'Surveilans ISPA Berat Indonesia' (SIBI) enabled the ARI Subdirectorate to focus on the epidemiology and influenza disease control program aspects whilst NIHRD focused on the laboratory diagnostics. The objectives of SIBI were to monitor epidemiological and virological trends of influenza in different parts of Indonesia. Even though the system may detect cases of novel or emerging influenza viruses, this was not deemed the primary objective of the system as other systems such as the nationally comprehensive Early Warning Alert and Response System (EWARS) provide such function.

One aspect that had to be considered carefully in establishing SIBI was the selection of the geographic locations and hospitals to become sentinel sites. The WHO guideline highlights system stability, feasibility and representativeness as the most important factors to consider when choosing a sentinel site [6]. The guideline also suggests considering the suitability of sites to assess the disease burden of influenza whereby information about the population served by the hospital can be determined to enable disease incidence rate calculation. When multiple sentinel sites are being considered, WHO recommends selecting sites that represent different population centers or climate zones since this will provide information about transmission patterns among sub-populations with unique demographic or socio-economic characteristics. Critically, the guideline states that there is no ideal number of sentinel sites in a country but that countries should start with one or a few sentinel sites and only expand if these function well.

As Indonesia is a large archipelago nation, national decision-makers are cognizant of the regional differences in culture, religion, economic prosperity and development [8]. This results in a push for public health initiatives to have representation according to administrative divisions such as a surveillance site in each province or to apply statistical approaches for calculation of the number of sentinel sites. These approaches may not be suited or feasible for sentinel surveillance systems where the objective is to obtain high-quality patient level data to monitor regional clinical, epidemiological and microbiological disease trends. For example, having sentinel sites in contiguous provinces with similar population demographics would yield more cases enrolled into the system but would not yield additional novel information about regional variation in disease activity. Similarly, having representativeness according to population density would yield large numbers of sentinel sites due to the size of the country but would also not necessarily capture unique regional characteristics. Lastly, since influenza surveillance involves costly laboratory diagnostic testing, the larger the number of sentinel sites the more expensive the shipment and testing of specimens.

Considering the guidance from WHO and the national prerogatives, deciding on the number, location and hospitals to be selected as sentinel SIBI sites was a challenge. Previous studies have reported on lessons learnt from establishing influenza surveillance systems $[9,10]$, but none have reported comprehensively on the process of sentinel site selection. This study describes 
Table 1 Indonesia's nationally-administered surveillance systems for acute respiratory infections, pneumonia and influenza, as of September 2012

\begin{tabular}{|c|c|c|c|c|c|c|c|}
\hline MOH system administrator & Surveillance system & General objective & Type & Sites & $\begin{array}{c}\text { Starting } \\
\text { year }\end{array}$ & $\begin{array}{l}\text { Reporting } \\
\text { frequency }\end{array}$ & $\begin{array}{l}\text { Laboratory } \\
\text { testing }\end{array}$ \\
\hline ARI subdirectorate & $\begin{array}{l}\text { Sentinel pneumonia } \\
\text { surveillance* }\end{array}$ & Monitor trends of pneumonia & Sentinel, aggregated data & $\begin{array}{l}40 \text { health centers and } \\
40 \text { district hospital }\end{array}$ & 2007 & Monthly & No \\
\hline ARI subdirectorate & $\begin{array}{l}\text { Routine pneumonia } \\
\text { surveillance }\end{array}$ & $\begin{array}{l}\text { Program performance for } \\
\text { pneumonia case detection }\end{array}$ & Population-based, aggregated data & All health centers & 1983 & Monthly & No \\
\hline Surveillance subdirectorate & $\begin{array}{l}\text { Early warning alert and } \\
\text { response system }\end{array}$ & Outbreak detection & Population-based, aggregated data & All health centers & 2008 & Weekly & No \\
\hline Surveillance subdirectorate & $\begin{array}{l}\text { Monthly outbreak-prone } \\
\text { disease surveillance }\end{array}$ & Trends for notifiable diseases & Population-based, aggregated data & $\begin{array}{l}\text { All health centers and } \\
\text { some district hospitals }\end{array}$ & 2003 & Monthly & No \\
\hline Surveillance subdirectorate & ILI syndromic surveillance & Outbreak detection & Sentinel, aggregated data & 20 Health Centers & 2010 & Weekly & No \\
\hline $\mathrm{NIHRD}$ & ILI virological surveillance & Influenza virus trends & Sentinel, case-based & 26 Health Centers & 2006 & Weekly & Yes (influenza) \\
\hline NIHRD & SARI virological surveillance* & Viral and bacterial trends & Sentinel, case-based & 10 tertiary hospitals & 2006 & Monthly & $\begin{array}{l}\text { Yes (viral and bacterial } \\
\text { pathogens) }\end{array}$ \\
\hline
\end{tabular}

MOH Ministry of Health, ILI Influenza-like IIIness; SARI Severe Acute Respiratory Infection, ARI Acute Respiratory Infection, NIHRD National Institutes for Health Research and Development.

* Systems were terminated in December 2012 and resources combined to create Surveilans ISPA Berat di Indonesia (SIBI).

\#Number of district hospitals participating is unknown because hospital participation is not mandated nationally. 
the process and rationale by which the six hospitals were selected as sentinel SIBI sites in Indonesia. The experience may guide other countries interested in adopting the WHO guideline and provide an approach for sentinel site selection or future surveillance system expansion.

\section{Methods}

\section{Step one: creation of a hospital shortlist}

Indonesia has 2,165 public and private hospitals [11]. Before applying the $\mathrm{WHO}$ guideline for sentinel site selection, we shortlisted from 2,165 hospitals to a manageable number to enable detailed assessment and final selection. To achieve this, we limited selection to public hospitals that had previously participated in the sentinel pneumonia or SARI surveillance systems. We ranked these 50 hospitals in terms of two performance indicators, timeliness and completeness of data reporting to national level. Ranking of hospitals according to their surveillance performance history enabled prioritization of good sites in subsequent steps of the selection process. Timeliness indicated the proportion of on-time reports received by $\mathrm{MOH}$ from the sentinel site, where $100 \%$ indicated full compliance with data reporting requirements (by the 10th of each month). Completeness indicated the number of reports made to $\mathrm{MOH}$ per year, where $100 \%$ indicated full compliance (12 reports received from the sentinel site per year). Timeliness and completeness data for the 50 hospitals were extracted from the $\mathrm{MOH}$ surveillance system databases. Hospitals with less than $50 \%$ timeliness and completeness were excluded based on poor surveillance performance history. This resulted in 20 hospitals in the shortlist from Step One (Figure 1).

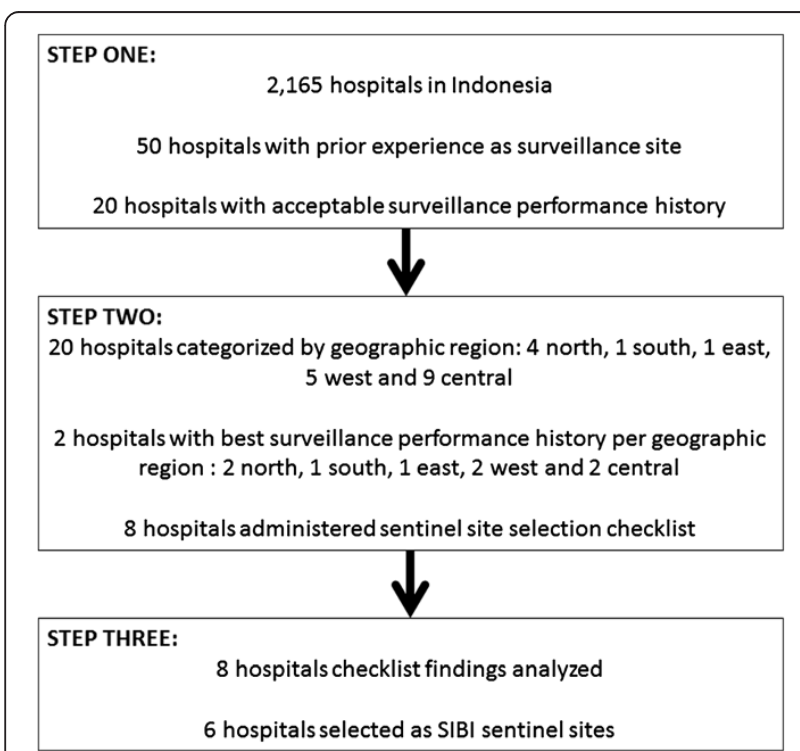

Figure 1 Three-step process for SIBI sentinel site selection.

\section{Step two: application of WHO guideline}

The second step was to operationalize the WHO recommended attributes for sentinel site selection; stability, representativeness and feasibility. Since influenza disease burden has not been established in Indonesia, we incorporated this into the selection process.

For stability, the WHO guideline recommended calculating the funds needed to cover general costs of surveillance operations for the long term. We operationalized this by calculating the yearly costs of one sentinel site and determining the number of sites that could be established using funds available from $\mathrm{MOH}$ and donors for the next three years. Cost calculations were based on previous surveillance system experience and included one training for five hospital staff ( 2 doctors, 1 nurse, 1 laboratory worker and 1 medical records staff), four monitoring visits to the site, six-monthly national meetings and costs for stationery and staff incentives. Based on previous SARI surveillance experience, each sentinel site was expected to generate 150 specimens per year. Costs for specimen collection, shipment to NIHRD and testing using real-time polymerase chain reaction (RT-PCR) and virus isolation (for $10 \%$ of specimens) were also calculated.

For representativeness, we first considered geographic representation of the hospitals. We ranked the 20 hospitals from Step One according to their geographic location to represent west (Sumatera island, $n=5$ ), north (Sulawesi island, $\mathrm{n}=4$ ), central (Java and Kalimantan islands, $\mathrm{n}=9$ ), south (East and West Nusa Tenggara islands, $\mathrm{n}=1$ ) and east (Maluku and Papua islands, $\mathrm{n}=1$ ). For hospitals in the same geographic regions, only the two top-performing hospitals were kept in the list and the others excluded. This resulted in a shortlist of eight hospitals - a number more aligned with the resources available for the surveillance system (Figure 1).

We developed a sentinel site selection checklist to address the other attributes and criteria. This included five questions to assess representativeness, such as the type of hospital (general versus specialty), accessibility of wards for influenza surveillance and the demographics of the population served by the hospital (Table 2). For feasibility, we included 13 questions to assess the motivation of the hospital to participate in the system, the infrastructure and human resource capacity available for surveillance activities (Table 2).

As stated by the WHO guideline, adequate patient volumes and ability to ascertain the hospital's catchment population are needed to calculate influenza disease burden. We added three questions to the checklist to explore these issues. For patient volumes, we requested data on total patient admissions not respiratory patient admissions since hospitals may vary in their disease coding and recording systems. 
Table 2 Checklist criteria and findings from the eight hospitals visited for sentinel site selection

\begin{tabular}{|c|c|c|c|c|c|c|c|c|c|}
\hline Attribute & Criteria & Maluku & $\begin{array}{l}\text { North } \\
\text { Sulawesi }\end{array}$ & $\begin{array}{l}\text { East } \\
\text { Kalimantan }\end{array}$ & $\begin{array}{l}\text { South } \\
\text { Kalimantan }\end{array}$ & $\begin{array}{l}\text { North } \\
\text { Sumatra }\end{array}$ & Yogyakarta & $\begin{array}{l}\text { West Nusa } \\
\text { Tenggara }\end{array}$ & $\begin{array}{l}\text { West } \\
\text { Kalimantan }\end{array}$ \\
\hline \multirow[t]{13}{*}{ Feasibility } & $\begin{array}{l}\text { 1. Hospital management agreeable to influenza } \\
\text { surveillance }\end{array}$ & Yes & Yes & Yes & No & Yes & Yes & Yes & No \\
\hline & 2. Two surveillance coordinating doctors available & Yes & Yes & Yes & Yes & Yes & Yes & Yes & Yes \\
\hline & 3. Surveillance nurse available to screen patients & Yes & Yes & Yes & Yes & Yes & Yes & Yes & Yes \\
\hline & $\begin{array}{l}\text { 4. Surveillance laboratory staff available for specimen } \\
\text { collection }\end{array}$ & Yes & Yes & Yes & Yes & Yes & Yes & Yes & Yes \\
\hline & $\begin{array}{l}\text { 5. Surveillance medical records staff available to } \\
\text { report data }\end{array}$ & Yes & Yes & Yes & Yes & Yes & Yes & Yes & Yes \\
\hline & 6. Refrigerator for specimen storage available & Yes & Yes & Yes & Yes & Yes & Yes & Yes & Yes \\
\hline & 7. Specimen courier service available & Yes & Yes & Yes & Not sure & Not sure & Not sure & Yes & Yes \\
\hline & 8. Computer and internet access available & Yes & Yes & Yes & Yes & Yes & Yes & Yes & Yes \\
\hline & 9. Frequent internet problems at hospital & Yes & Yes & No & Yes & No & No & Yes & No \\
\hline & 10. Adequate power supply for refrigerator & Yes & Yes & Yes & Yes & Yes & Yes & Yes & Yes \\
\hline & 11. Back up generator available & Yes & Yes & Yes & Yes & Yes & Yes & Yes & Yes \\
\hline & $\begin{array}{l}\text { 12. Electronic or paper record for medical } \\
\text { record system }\end{array}$ & Electronic & Paper & Paper & Electronic & Electronic & Electronic & Paper & Electronic \\
\hline & 13. ICD 10 used & Yes & Yes & Yes & Yes & Yes & Yes & Yes & Yes \\
\hline \multirow[t]{5}{*}{ Representativeness } & 14. District or province level hospital & Province & District & Province & District & District & District & Province & District \\
\hline & 15. Hospital for all age groups & Yes & Yes & Yes & Yes & Yes & Yes & Yes & Yes \\
\hline & 16. Hospital for all socioeconomic level populations & Yes & Yes & Yes & Yes & Yes & $70 \%$ low income & $60 \%$ low income & Yes \\
\hline & 17. All wards can participate in surveillance & Yes & Yes & Yes & Yes & Yes & Yes & Yes & Yes \\
\hline & 18. General or specialty hospital & General & General & General & General & General & General & General & General \\
\hline \multirow[t]{3}{*}{ Disease burden calculation } & 19. Hospital patient admissions per month & 1100 & 576 & 1200 & 1000 & 240 & 920 & 1100 & 590 \\
\hline & $\begin{array}{l}\text { 20. Patient home addresses available so that hospital } \\
\text { catchment population can be estimated }\end{array}$ & Yes & Yes & Yes & Yes & Yes & Yes & Yes & Yes \\
\hline & $\begin{array}{l}\text { 21. X-ray conducted routinely for respiratory disease } \\
\text { patients }\end{array}$ & Not sure & No & No & No & Yes & No & No & No \\
\hline
\end{tabular}


Four teams comprising ARI Subdirectorate, NIHRD and Centers for Disease Control and Prevention (US-CDC) staff were trained in the sentinel site selection checklist objective and administration. Each team visited two hospitals where the teams met with the hospital director (or management representative), the medical and nursing staff and the laboratory team. These hospital personnel were targeted as they could answer the various administrative and procedural questions in the checklist. After introducing the concept of SIBI and answering any general questions, one team member administered the questionnaire and the others documented the answers. The teams then toured the hospital facilities including the medical records unit, emergency/patient admission, the laboratory and some patient wards. This enabled visual confirmation of the hospital capacity and likely flow of SARI patient enrolment into SIBI. The teams also reviewed medical records data on the number of monthly hospital admissions. Each sentinel site selection visit took 4-6 hours and all hospitals were visited in a three-week period. Funding for the sentinel site selection visits was made available by WHO.

\section{Step three: analysis and selection of sentinel sites}

Checklist data from the eight hospitals were entered into a spreadsheet and analyzed descriptively (Table 2). The four teams met to determine which hospitals would be enrolled as SIBI sentinel sites based on the visit findings. Hospital management motivation to participate as a surveillance site was deemed a key criterion for selection as solutions to other issues could be addressed as long as the hospital was willing to collaborate. Thus, if hospital management did not welcome the surveillance activity, it no longer qualified for selection.

\section{Results and discussion Stability}

The cost of establishing one sentinel site was USD 40,000 per year. Costs included training for five hospital staff (USD 5,900), specimen management and testing (USD 8,700), quarterly national monitoring missions by ARI Subdirectorate and NIHRD staff (USD 10,100), six-monthly national review meetings (USD 9,300) and administrative costs such as printing forms, hospital staff incentive payments, stationery and part-salary of a national level data manager apportioned to one-third of the person's time (USD 5,580). From the MOH government and donor funds available and projected for the next three years, six SIBI sentinel sites could be established.

\section{Representativeness}

Based on the geographic location of the eight hospitals visited, there was representation of east (Maluku), west (North Sumatra), north (North Sulawesi), central (Yogyakarta, Kalimantan) and south Indonesia (West Nusa Tenggara).
All hospitals were general hospitals and reported coverage of all age-groups and socio-economic population groups, although the hospitals in West Nusa Tenggara and Yogyakarta reported that more than $50 \%$ of their hospital admissions were for low socioeconomic population (Table 2). All hospitals confirmed that surveillance could be conducted in all wards where SARI patients may be admitted.

\section{Feasibility}

All eight hospitals visited had five staff available for the day-to-day operation of SIBI surveillance as well as basic infrastructure requirements such as a refrigerator, back-up power supply, computers and internet service for data reporting (Table 2). Even though four sites reported unreliable internet service at the hospital, back-up measures such as a WIFI modem could be utilized. For specimen shipment to NIHRD in Jakarta, three sites were uncertain of couriers available in the hospital vicinity. However, based on the locations of these hospitals and the NIHRD team's experience, courier services were likely to be available. Two hospitals did not express willingness to participate in the surveillance system due to difficulties to internally coordinate surveillance activities (Table 2).

\section{Disease burden}

All hospitals collected patient home addresses which would enable estimation of the hospital catchment population (Table 2). Six hospitals had >500 admissions per month and two reported lower admission rates (220 and 240 admissions per month for West Nusa Tenggara and North Sulawesi respectively). None of the hospitals had a standard protocol mandating chest $\mathrm{x}$-rays for respiratory patients, but all hospitals estimated that 50\% of patients would have $\mathrm{x}$-rays requested by the treating physician. For surveillance purposes, the number of patients with $\mathrm{x}$-rays generated through this approach would be large enough to enable analysis trends relating to pneumonia. Thus, for future calculation of disease burden of influenza in Indonesia, all eight hospitals were considered suitable.

\section{Conclusion}

Six hospitals were selected and enrolled as SIBI sentinel sites based on the multi-step sentinel site selection process (Figure 1). These were the hospitals in Maluku (east), North Sulawesi (north), East Kalimantan (central), North Sumatra (west), Yogyakarta (central) and West Nusa Tenggara (south, Figure 2). The lack of hospital management motivation to participate as a sentinel site was the key reason for not selecting the hospitals in South and West Kalimantan.

The WHO guideline was useful as it identified the key attributes to consider in sentinel site selection and it 


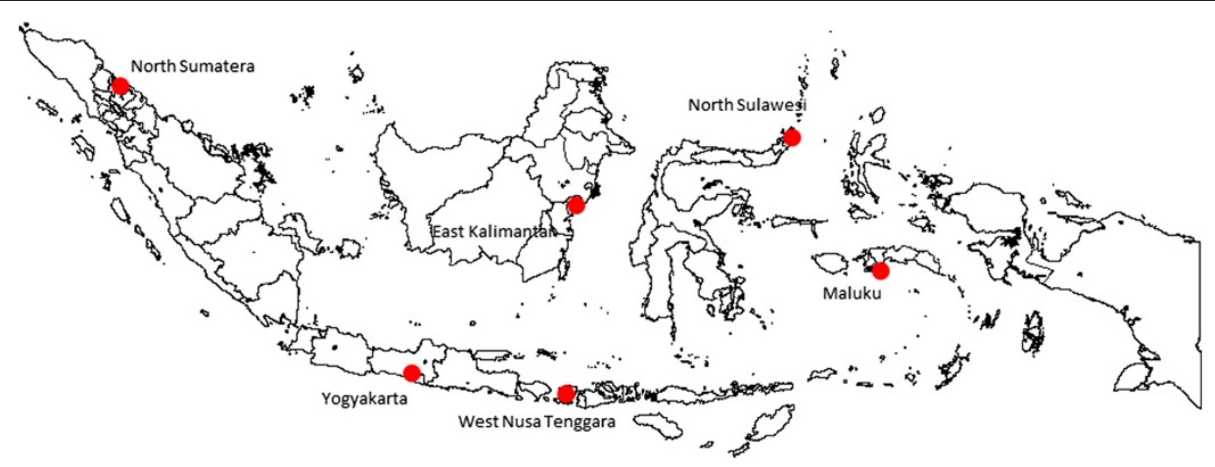

Figure 2 Location of six SIBI sites established in Indonesia, 2013.

helped address public health decision maker concerns regarding the geographic representation of the surveillance system. However, since the WHO guideline does not provide an algorithm or scoring system for site selection, we had to operationalize the attributes into quantitative and qualitative criteria, and to prioritize these according to our context. We also had to develop our own approach for shortlisting from all the hospitals in Indonesia to a number that could be assessed using the detailed WHO approach.

Despite using a multi-step approach to sentinel site selection, there were some limitations to our process. These included reliance on self-report for checklist items such as the hospital's motivation to participate in the surveillance system and availability of hospital staff to conduct surveillance activities. Respondents may have sought to appease the assessment team by positively biasing their answers. To address this limitation, hospitals ultimately selected as sentinel sites were asked to write a formal letter to confirm their commitment to become a surveillance site, and to list surveillance staff and their specific role in the surveillance system. For other checklist items, such as the proportion of respiratory disease patients receiving chest $x$-ray or the socioeconomic level of the population served by the hospital, it was not feasible to verify the answers provided by the hospital team during the assessment visit. However, these issues will be revisited in more detail once the surveillance system stabilizes and disease burden can be enumerated. Another limitation is that the sentinel site selection process does not guarantee good surveillance performance. We endeavor to maximize performance through the routine monitoring visits and twice-yearly review meetings.

As the primary objective of SARI surveillance is to monitor the epidemiological and virological characteristics of influenza, surveillance does not have to be comprehensive at all hospitals or in all provinces. A few sentinel sites may appropriately represent the situation in-country and to inform public health officials on groups that need to be targeted for preventive and treatment measures. To date, there have been no studies to investigate whether influenza patterns vary across regions in Indonesia, but since difference regions have different climatic patterns and this is known to impact influenza circulation [12,13], it is possible that influenza patterns are different. Future studies will be required to determine whether the six SIBI sites adequately represent influenza activity in Indonesia and the extent to which the clinical, epidemiological and virological findings from the six sentinel sites are generalizable. This approach may address decision-maker concerns about surveillance representativeness in a cost-efficient manner.

As SIBI stabilizes over time and long term resources are assured, there is potential to enhance the system to also monitor other important diseases such as dengue, meningitis and severe diarrheal illness [6]. This allows for further efficiencies in utilization of sentinel surveillance systems for specific public health objectives and to minimize the burden of establishing additional standalone systems. The surveillance system can also be used to evaluate the benefits of public health interventions and to monitor disease severity and impact during a future influenza pandemic [14]. SIBI can also be used for emerging zoonotic diseases and allow for laboratorybased collaborations with the Ministry of Agriculture on diseases such as Nipah and Hendra virus or emerging coronavirus infections. Overall, we hope that this report clarifies the rationale for the geographic distribution, the number of sites and the methods for selection.

\section{Competing interests}

The authors declare that they have no competing interests.

Authors' contributions

NKS, MS, CYP, ODS, AB, RR and GS conceptualized and designed the study. NKS, MS CYP, EZ, AF, A, HA, EH and GS collected, analyzed and interpreted the data. GS wrote the first draft of the manuscript. All authors participated in drafting or revising the manuscript for intellectual content and all provided final approval for its publication. 


\section{Acknowledgements}

We acknowledge Ministry of Health decision makers at the National Institute of Health Research and Development and the Directorate-General for Disease Control and Environmental Health for their leadership and the commitment of the various hospitals participating as sentinel sites.

\section{Author details}

${ }^{1}$ National Institute of Health Research and Development, Ministry of Health, Jakarta, Indonesia. ${ }^{2}$ Disease Control and Environmental Health, Ministry of Health, Jakarta, Indonesia. ${ }^{3}$ Influenza Division, United States Centers for Disease Control and Prevention, Jakarta, Indonesia.

Received: 23 December 2013 Accepted: 15 September 2014 Published: 23 September 2014

\section{References}

1. Cannell JJ, Zasloff M, Garland CF, Scragg R, Giovannucci E: On the epidemiology of influenza. Virol J 2008, 5:29.

2. Clark NM, Lynch JP III: Influenza: epidemiology, clinical features, therapy, and prevention. Semin Respir Crit Care Med 2011, 32(4):373-392.

3. MMWR Morb Mortal Wkly Rep: 2009 pandemic influenza A (H1N1) virus infections - Chicago, Illinois, April-July 2009. MMWR Morb Mortal Wkly Rep 2009, 58(33):913-918.

4. Kelly PM, Kotsimbos T, Reynolds A, Wood-Baker R, Hancox B, Brown SG, Holmes M, Simpson G, Bowler S, Waterer G, Irving LB, Jenkins C, Thompson PJ, Cheng AC: FluCAN 2009: initial results from sentinel surveillance for adult influenza and pneumonia in eight Australian hospitals. Med J Aust 2011, 194(4):169-174.

5. Ong JB, Chen MI, Cook AR, Lee HC, Lee VJ, Lin RT, Tambyah PA, Goh LG: Real-time epidemic monitoring and forecasting of H1N1-2009 using influenza-like illness from general practice and family doctor clinics in Singapore. PLoS One 2010, 5(4):e10036.

6. WHO interim global epidemiological surveillance standards for influenza. www.who.int/influenza/resources/documents/influenza_surveillance_manual/en/.

7. WHO recommended surveillance standards. http://www.who.int/csr/ resources/publications/surveillance/WHO_CDS_CSR_ISR_99_2_EN/en/.

8. Statistics Indonesia. http://www.bps.go.id/.

9. Kebede S, Conteh IN, Steffen CA, Vandemaele K, Wurie I, Alemu W, Kuti-George F, Dafae F, Jambai A, Yahaya AA, Kasolo FC: Establishing a national influenza sentinel surveillance system in a limited resource setting, experience of Sierra Leone. Health Res Pol Syst 2013, 11(22):1-8.

10. Steffen C, Debellut F, Gessner BD, Kasolo FC, Yahaya AA, Ayebazibwe N, Bassong O, Cardoso Y, Kebede S, Manoncourt S, Vandemaele KA, Mounts AW: Improving influenza surveillance in sub-Saharan Africa. Bull World Health Organ 2012, 90:301-305.

11. Indonesia Hospitals. http://202.70.136.52/rsonline/report/report_by_catrs.php.

12. Chan PK, Mok HY, Lee TC, Chu IM, Lam WY, Sung JJ: Seasonal influenza activity in Hong Kong and its association with meteorological variations. $J$ Med Virol 2009, 81(10):1797-1806.

13. Tang JW, Lai FY, Nymadawa P, Deng YM, Ratnamohan M, Petric M, Loh TP, Tee NW, Dwyer DE, Barr IG, Wong FY: Comparison of the incidence of influenza in relation to climate factors during 2000-2007 in five countries. J Med Virol 2010, 82(11):1958-1965.

14. World Health Organization: Assessing the Severity of an Influenza Pandemic. Geneva: 2009. http://www.who.int/csr/disease/swineflu/assess/ disease_swineflu_assess_20090511/en/.

\section{Submit your next manuscript to BioMed Central and take full advantage of:}

- Convenient online submission

- Thorough peer review

- No space constraints or color figure charges

- Immediate publication on acceptance

- Inclusion in PubMed, CAS, Scopus and Google Scholar

- Research which is freely available for redistribution

Submit your manuscript at www.biomedcentral.com/submit 